\section{P336 THE SEXUAL HEALTH KNOWLEDGE, ATTITUDES AND BEHAVIOUR OF WOMEN LIVING WITH HIV IN BANTEN PROVINCE, INDONESIA}

Dyah Juliastuti ${ }^{*}$, Judith Dean, Lisa Fitzgerald. The University of Queensland, School of Public Health, Herston, Australia

\subsection{6/sextrans-2019-sti.444}

Background Among certain communities and nations, the sexual health rights of women living with HIV (WLHIV) are often neglected and considered less important. Social, cultural, and religious believes may restrict safer sexual practices. This study examines the sexual health knowledge, attitude, and practices of WLHIV in Banten Province, Indonesia, and the factors influencing these practices.

Methods A cross-sectional written survey of 209 WLHIV aged 18 to 50 years from seven Banten Province regions, was conducted between May and November 2017 using five peer recruiters. Descriptive and binary regression analysis were performed.

Results The majority were Muslim (87.6\%), married (58.9\%), and high-school graduated (88.6\%). 31.4\% experienced intimate partner violence (IPV). $77 \%$ had some knowledge of sexually transmitted infection (STI), sourcing information from health care providers (56.9\%) and the internet (23.0\%). Most believed that a woman could refuse sexual intercourse with their partner because of postpartum/menstruation (78.9\%), tiredness/unwillingness (67.7\%), partner's STI (64.1\%), and his infidelity (62.7\%). However, only $49.3 \%$ believed that they might refuse sex if the partner opposed condom use. Of the 171 sexually active participants, $78.4 \%$ had only one sexual partner. Condoms were the primary contraceptive used; however, $68.4 \%$ reported inconsistent condom use which was significantly associated with women's inability to buy condoms (aOR6.54, 95\%CI:2.29-18.74), negotiate condom use (aOR6.11\%CI:1.69-22.15), and being unmarried (aOR3.04, 95\%CI:1.14-8.13). 33.3\% self-reported a history/symptoms of STI which was significantly associated with new HIV-diagnosis $(\mathrm{aOR}=4.53, \quad 95 \% \mathrm{CI}=2.07-9.91), \quad$ multiple sex-partners $(\mathrm{aOR}=4.25,95 \% \mathrm{CI}=1.73-10.43)$, lack of internet exposure $(\mathrm{aOR}=4.24, \quad 95 \% \mathrm{CI}=1.55-11.55), \quad \mathrm{IPV} \quad(\mathrm{aOR}=3.04, \quad 95 \%$ $\mathrm{CI}=1.22-7.55)$, or unprotected sex $\quad(\mathrm{aOR}=2.97, \quad 95 \%$ $\mathrm{CI}=1.33-6.60)$.

Conclusion The sexual health of WLHIV in Indonesia is affected by their relationship status, access to condoms, and ability to negotiate their use. Strategies focused on building a women's capability and support to defend their sexual rights are needed to improve the women's health, facilitate choice and reduce risk of onward HIV transmission.

Disclosure No significant relationships.

\section{P337 A STUDY ON HOW SEXUAL EMPOWERMENT UNDER MIXED-GENDER DYADS INFLUENCES SEXUAL HEALTH AMONG WOMEN IN INDIA}

Raman Mishra*. International Institute for Population Sciences, Population Studies, Mumbai, India

\subsection{6/sextrans-2019-sti.445}

Background Sexually transmitted infections (STIs) are a key reproductive and public health concern worldwide. STIs are pernicious players in the global burden of disease. According to The World Health Organization estimates, each year there are an estimated 357 million new infections of STIs globally. In India, the prevalence of STIs among women in the union of reproductive age remained constant at 11 percentage points from NFHS-3 to NFHS-4. This number highlights the gravity of the unwavering situation of poor reproductive health among women in India.

Methods This study used data from the National Family Health Survey (NFHS-4) conducted in 2015-16. It included questions on demographic and socioeconomic characteristics of individuals, their sexual behavior, gender relations and whether they had suffered from an STI in the 12 months prior to the survey i.e. self-reported STI. Multiple logistic regression models were fitted to determine the relationship between predictors and the reported STI status.

Results Sexual empowerment was significantly associated with STI status, but odds of reporting STIs were higher among sexually empowered women. Women's participation in decisionmaking concerning their own health reduced the odds of contracting STIs. The strongest predictors of reporting STIs, namely sexual violence and the number of lifetime partners, are at direct risk of STIs. STIs was also positively associated with partners' controlling behaviors. With respect to STIs, sexual empowerment does not appear to protect women in the union who have violent controlling partners. Sexual empowerment is also not protective where a woman having multiple partner.

Conclusion Interventions promoting sexual health must effectively address roles that perpetuate unhealthy sexual behaviors and relations within the union. It is therefore imperative to promote fidelity and better communication between partners in the union and necessary to encourage regular screening and treatment of STIs. Sexual decisions must be subject to joint opinions in order to achieve and secure complete reproductive health and rights.

Disclosure No significant relationships.

\section{P338 PREVALENCE AND MUCOSAL IMPACT OF STIS IN YOUNG WOMEN FROM MOMBASA, KENYA WITH VARYING EXPOSURE TO SEX WORK}

${ }^{1}$ Ruth Mwatelah, ${ }^{2}$ Shelley Peterson, ${ }^{2}$ Christine Bonner, ${ }^{1}$ Tosin Omole, ${ }^{1}$ Faisal Nuhu, ${ }^{1}$ Naima Jahan, ${ }^{3}$ Nzioki King'Ola, ${ }^{3}$ Sammy Wambua, ${ }^{3}$ Peter Gichangi, ${ }^{4}$ Eve Cheuk, ${ }^{2}$ Grant Mcclarty, ${ }^{5}$ Irene Martin, ${ }^{6}$ Marissa Becker, ${ }^{7}$ Sharmistha Mishra, ${ }^{1}$ Lyle Mckinnon* 'University of Manitoba, Department of Medical Microbiology and Infectious Diseases, Winnipeg, Canada; ${ }^{2}$ National Microbiology Lab (NML), Public Health Agency of Canada (PHAC), Winnipeg, Canada, Winnipeg, Canada; ${ }^{3}$ International Centre for Reproductive Health-Kenya, Mombasa, Kenya; ${ }^{4}$ Centre for Global Public Health, Department of Community Health Sciences, Winnipeg, Canada; ${ }^{5}$ National Microbiology Laboratory, Winnipeg, Canada; ${ }^{6}$ University of Manitoba, Centre for Global Public Health, Department of Community Health Sciences, Winnipeg, Canada; 'St. Michael's Hospital, Li Ka Shing Knowledge Institute, Toronto, Canada

\subsection{6/sextrans-2019-sti.446}

Background Bacterial STIs increase mucosal inflammation and HIV acquisition risk. However, most data are limited to symptomatic STIs, and more data are required regarding the prevalence and correlates of asymptomatic bacterial STIs among high-risk young women, and how these vary by exposure to sex work.

Methods We estimated the prevalence of 6 STIs in urine collected during a cross-sectional study of women aged 14-24 years in Mombasa, Kenya $(n=870)$. Participants were recruited from sex work hotspots, and self-identified as engaged or not 
engaged in formal sex work. STIs including Ureaplasma urealyticum (UU), Chlamydia trachomatis (CT), Mycoplasma hominis (MH), Trichomonas vaginalis (TV), Mycoplasma genitalium $(\mathrm{MG})$ and Neisseria gonorrhea (NG) were detected using the Seegene Anyplex qPCR panel. The cervicovaginal microbiome and cytokines were also characterized in a subset of 168 participants.

Results The prevalence of any STI was 25.1\% (95\% CI, 22.328.0\%). The most common STIs were UU (13.2\%) and $\mathrm{MH}$ (11.3\%), followed by CT (6.6\%), TV (3.2\%), NG (0.3\%), and MG (0.5\%). U. urealyticum was more prevalent among sex workers compared to non-sex workers (19.7 vs $10.4 \%$, $\mathrm{p}=0.001)$. Number of partners positively correlated with UU $(\mathrm{P}=0.004)$, while duration of sexual activity was positively correlated with $\mathrm{MH}(\mathrm{P}<0.001)$ and UU $(\mathrm{P}=0.023)$. Recent history of STI treatment did not correlate with any STI. $\mathrm{MH}$ was most associated with symptoms, including vaginal discharge, itching, and painful urination $(\mathrm{P}<0.001)$. All STIs except CT were positively correlated with pro-inflammatory cytokines. UU and $\mathrm{MH}$ were correlated with vaginal microbiota alpha diversity $(\mathrm{P}<0.001)$.

Conclusion $U$. urealyticum and $M$. hominis were the most prevalent STIs among young women from Mombasa, Kenya, and these organisms were associated with sexual exposure and an inflammatory mucosal milieu that has been linked to increased HIV acquisition.

Disclosure No significant relationships.

\section{P339 FACTORS OF RISKY SEXUAL BEHAVIOR AMONG LONG- HAUL TRUCKERS IN A SOUTHERN NIGERIAN TOWN}

\footnotetext{
${ }^{1}$ Paul Okojie*, ${ }^{2}$ Kingsley Okafor, ${ }^{3}$ Omoyemen Bello, ${ }^{3}$ Esohe Ogboghodo, ${ }^{3}$ Abimbola Adesanya. 'Liberty University, Public and Community Health, Lynchburg, USA; ${ }^{2}$ Health Research and Policy Development Foundation, Abuja, Nigeria; ${ }^{3}$ University of Benin, Community Health, Benin City, Nigeria
}

10.1136/sextrans-2019-sti.447

Background Long-haul drivers play a role in the spread of sexually transmitted infections (STI) globally. Truck drivers engage in frequent travel away from home. They are exposed to risky sex with commercial sex workers who are a reservoir of HIV and other STIs. The study aimed to identify factors associated with commercial sexual exposure among long-haul truckers in a southern Nigerian town.

Methods A sample of 300 long-haul truckers from a highway park in Ahor, southern Nigeria was recruited in a cross-sectional study. An interviewer-administered questionnaire was used to collect socio-demographic and sexual risk behavior data. Data were analyzed with SPSS version 20 software. Chisquare test, Odds ratio, and confidence intervals were used to find the association between condom use, STI history, HIV status, duration of a trip, number of sexual partners, and truckers' exposure to commercial sex workers.

Results While $42.0 \%$ of the truckers used condoms, $58.0 \%$ were non-users. Reasons for non-condom use were commitment to partner (72.0\%), lack of pleasure (21.0\%), and beliefs (7.0\%). Fifty percent of respondents reported two or less sexual contacts while a third of them reported 12 sexual contacts in 6 months. Overall, $59.0 \%$ of respondents had a history of multiple sexual partners. Factors associated with truckers' exposure to a commercial sex worker were use of psychoactive substance (OR 2.5 (1.5-4.4), $p=0.00$; condom use (OR 16.3 (8.0-32.5), $p=0.00$; previous sexually transmitted infection (OR $2.5(1.2-5.1), p=0.01$. The odds of single-sex partner exposure to a commercial sex worker were $10 \%$ less than the odds in multiple sex partner truckers, $p=0.00$.

Conclusion This study shows that psychoactive substances and condoms may be fueling exposure to commercial sex among truck drivers. Intervention programs should emphasize a single partner relationship, target substance users and highlight the potential impact of previous sexually transmitted infections on the risk of acquiring an HIV infection.

Disclosure No significant relationships.

\section{P340 TRENDS IN HIGH-RISK SEXUAL RISK BEHAVIORS IN THE UNITED STATES, 2002-2015}

Jami Leichliter, Patricia Dittus*, Sevgi Aral. CDC, Atlanta, USA

\subsection{6/sextrans-2019-sti.448}

Background Reportable STIs have largely been increasing in the United States; yet, few national-level studies have examined potential reasons for the increase. We used data from the 2002 and 2011-15 National Survey of Family Growth to examine trends in high-risk sexual behavior among key subpopulations with disproportionate STI rates.

Methods The subpopulations we examined included sexually active: men who have sex with men (MSM) and adolescent, young adult, Hispanic, and non-Hispanic black women who had sex with men (WSM) and men who had sex with women (MSW). High-risk sexual behavior (HRSB) was a composite variable (yes, no) that included endorsement of any of the following: giving or receiving money or drugs for sex or having a partner who was bisexual, non-monogamous, HIV-positive, or who injected drugs. We used weighted data to examine the prevalence of HRSB over time for each subpopulation and conduct logistic regressions adjusting for race/ethnicity, age, marital status, poverty and education.

Results HRSB either did not change or declined over time. Overall, among WSM, reported HRSB significantly declined over time (15.1\% in 2002 and 12.4\% in 2011-15). Among men, we also found significant declines in reported HRSB for MSW (15.4\% in 2002 and $12.2 \%$ in 2011-15) and MSM $(25.9 \%$ in 2002 and $13.1 \%$ in $2011-15)$. In subgroup analyses, there were more declines among WSM than MSW or MSM. However, in adjusted analyses, we found significant declines in reported HRSB, comparing 2002 to 2011-15, among non-Hispanic black $(\mathrm{aOR}=0.78,0.65-0.95)$ and adolescent $(\mathrm{aOR}=0.64,0.50-0.95) \quad \mathrm{WSM} ;$ Hispanic $(\mathrm{aOR}=0.49$, $0.35-0.67)$ and adolescent $(\mathrm{aOR}=0.57,0.41-0.79) \mathrm{MSW}$; and all MSM $(\mathrm{aOR}=0.42,0.24-0.75)$.

Conclusion While STIs are increasing, HRSB was steady or declined among key subpopulations with disproportionate STI rates. Results from adjusted analyses suggest these findings are not the result of changing population demographics. Further research is needed to explain the STI increases.

Disclosure No significant relationships. 